\title{
EL RENACIMIENTO DEL SINDICALISMO SOCIALISTA EN LAS MINAS DE HUELVA DURANTE LAS POSTRIMERÍAS DE LA DICTADURA DE PRIMO DE RIVERA: ESTRATEGIA Y ÉXITO ORGANIZATIVO
}

\section{MIGUEL ÁNGEL COLLADO AGUILAR UNIVERSIDAD DE HUELVA}

RESUMEN: Se analiza la reaparición del sindicalismo minero en Huelva, y especialmente en la Cuenca Minera de Riotinto, durante los últimos momentos de la dictadura de Primo de Rivera, para lo que se recurre fundamentalmente a la documentación de la Rio Tinto Company Limited y se tienen en cuenta tanto el protagonismo de González Peña y Manuel Llaneza como las reacciones de la patronal minera ante la irrupción del socialismo en las minas.

PALABRAS CLAVE: Socialismo, UGT, Minas de Riotinto, Dictadura de Primo de Rivera, Sindicato Católico.

\section{THE REVIVAL OF SOCIALIST TRADE UNIONISM IN THE MINES OF HUELVA DURING THE END OF PRIMO DE RIVERA'S DICTATORSHIP: STRATEGY AND ORGANISATIONAL SUCCESS}

\begin{abstract}
The reappearance of mining unionism in Huelva, and especially in the Riotinto Mining Basin, during the last moments of Primo de Rivera's dictatorship is analysed, for which the documentation of the Rio Tinto Company Limited is used fundamentally and both the protagonism of González Peña and Manuel Llaneza and the reactions of the mining bosses to the irruption of socialism in the mines are taken into account.
\end{abstract}

KEYWORDS: Socialism; UGT; Riotinto Mines; Primo de Rivera Dictatorship; Catholic Union. 


\section{Antecedentes: el sindicalismo minero socialista en la Huelva de la década de 1910-} 1920

A pesar de ser cierto que el anarquismo apareció en la provincia de Huelva en las últimas décadas del s. XIX y que en los primeros años del siglo siguiente hubo algunos intentos socialistas por asentarse en la misma, no lo es menos que ni una ni otra corriente lograron fundar organizaciones duraderas hasta la década de 1910 ni que durante la mayor parte de la misma fue la UGT la que dominó en el movimiento obrero onubense ${ }^{1}$.

Sin lugar a dudas, la principal causa de que las organizaciones obreras no llegaran a asentarse hasta épocas tan tardías hay que buscarla en el carácter cuasi colonial que las empresas mineras imprimían a las relaciones laborales, como tendremos ocasión de ver.

Del mismo modo, el hecho de que Huelva sea una provincia periférica debió ser fundamental a la hora de que las entidades político-sindicales del obrerismo demoraran sus esfuerzos para lograr su implantación en ella, todo ello a pesar de que sus establecimientos mineros eran los más importantes del país y empleaban a varios miles de trabajadores.

En cualquier caso, no será hasta 1912 cuando el sindicalismo socialista ponga sus miras en Huelva y envíe a varios hombres a poner en pie una organización en las minas, destacando entre ellos Eladio Fernández Egocheaga o el mismo Agustín Marcos al que veremos más adelante, ya a finales de la dictadura de Primo de Rivera, desarrollando esta misma labor.

En aquella ocasión, lo que se puso en pie fue un sindicato de base múltiple que, además de la actividad reivindicativa en lo laboral, buscaba la mejora de la situación obrera mediante el establecimiento de socorros o cooperativas de consumo, que impulsaba la acción política a través de la presentación de candidaturas a las distintas elecciones y que propugnaba el establecimiento de cajas de resistencia para poder mantener a los huelguistas durante los periodos de lucha ${ }^{2}$.

Es decir, que más que de sindicatos en sí mismos, de lo que se trataba era de unas organizaciones que intentaban aunar la lucha política y la sindical al tiempo que pretendían establecer mecanismos de solidaridad horizontal para facilitar la vida inmediata de los obreros.

\footnotetext{
${ }^{1}$ La bibliografía referente al movimiento obrero en las minas onubenses durante la década de 1910 no es muy abundante, como tampoco lo es la calidad de algunos trabajos que abordan ese aspecto de la Historia de Huelva. Aun así, la obra de De Paz Sánchez ha paliado esta realidad y conviene ser reseñada. En ella nos basamos para la redacción de este apartado y a ella remitimos para ampliar lo dicho.

DE PAZ SÁNCHEZ, José Juan, Entre el puerto y la mina, 3 Vol., Servicio de Publicaciones de la Universidad de Huelva y Puerto de Huelva, 2014- 2018, 3 vols.

2 BAR, Antonio: La C.N.T. en los años rojos-del sindicalismo revolucionario al anarcosindicalismo, Akal, Madrid, 1981, p. 126.
} 
Más allá de esto, lo que conviene señalar es que de lo que hablamos es de unos sindicatos autónomos tanto en lo económico como en lo organizativo, lo que proporcionará a sus líderes una amplia capacidad de acción y contrastará con los que veremos más adelante, a finales de la dictadura y durante la II República.

Además, hay que indicar que los primeros dirigentes del sindicalismo socialista serán especialmente belicosos, sobre todo para con las compañías mineras. De hecho, tan pronto como pongan en pie los primeros sindicatos empezarán a menudear las huelgas, que se iniciarán en puntos concretos de la geografía onubense o un departamento minero y terminarán por extenderse, ya sea por la provincia o a todo un sector o centro de trabajo.

Como ejemplo, mencionaremos las continuas huelgas que se sucedieron en las minas de Riotinto en 1913 y que, iniciadas en abril por los cargadores de torales de cobre, se extendieron por toda la provincia y paralizaron la producción de mineral en varias ocasiones ${ }^{3}$.

En este contexto, el sindicalismo socialista sufrirá una profunda represión, tanto por parte de las empresas como por el Estado, que terminará por generarle una profunda crisis a raíz de la Huelga General Revolucionaria de 1917 y que hará que a finales de la década haya que hablar de una organización muy menguada en comparación con la de los primeros años.

Por otra parte, es de mencionar que los primeros sindicatos adeptos a la CNT aparecerán durante la segunda mitad de la década, que desde 1917 irán ganándole terreno al socialismo y serán los que dirijan las huelgas mineras de 1920 en Riotinto. Sin ánimo de extendernos sobre ellas, diremos que las mencionadas huelgas de 1920 fueron las más importantes del periodo y culminaron con una derrota definitiva tanto para los socialistas como para los propios libertarios, siendo además la sentencia de muerte para la unidad de acción que ambos sindicatos venían manteniendo en todo el país desde hacía tres años ${ }^{4}$.

De hecho, después de esta huelga puede hablarse de la desaparición del sindicalismo de una y otra tendencia en toda la provincia, aun habiendo excepciones, como la de Peña de Hierro, a las que nos referiremos más adelante.

En el caso de la CNT está claro que, además de por la mencionada derrota y la consiguiente represión, la causa del ostracismo habría que buscarla en la imposibilidad de reorganización que le imponía el hecho de estar prohibida por la dictadura de Primo de

\footnotetext{
${ }^{3}$ Sobre esta huelga véanse el primer volumen de la obra escrita por PÉREZ LÓPEZ, Juan Manuel: Sindicalismo minero en Huelva. La huelga de 1913 en Río Tinto como paradigma de acción colectiva desde los órganos de representación obrera, Asociación para el Desarrollo Rural Cuenca Minera de Riotinto, Minas de Riotinto, 2007.

${ }^{4}$ Optamos por citar dos textos "militantes" sobre el particular en pro de que el lector pueda hacerse una idea de las consecuencias de la huelga para el Pacto de unidad sindical desde las dos perspectivas.

PIERATS, José: La CNT en la revolución española. Tomo 1, Eds. Carvajal: Colombia, 1988, pág. 30.

DEL ROSAL, Amaro: Historia de la UGT de España.1901-1939. Vol. 1, Eds. Grijalbo: Barcelona, págs. 228-235.
} 
Rivera, mientras que en el de la UGT quizás esté en que había dejado de ser útil para los trabajadores y la idea de mantener viva una organización incapaz de proporcionarles soluciones a sus problemas inmediatos era, cuanto menos, poco atractiva.

No en vano, esta última situación cambiará a finales de la dictadura de Primo de Rivera y, gracias a ello, veremos cómo se producirá un auténtico renacimiento del sindicalismo socialista en las minas de Huelva. En cuanto a la CNT como tal, no volverá a aparecer hasta los primeros momentos de la II República, todo ello a pesar de que en lo que sigue podremos ver cómo algunos de sus adeptos seguían presentes en las minas e incluso criticaban la acción de los reorganizadores del socialismo.

\section{El marco general: las elecciones al Comité Paritario de la minería}

Como es sabido, una de las prioridades de la dictadura primoriverista fue construir un Estado corporativo y, para hacerlo, puso en marcha unos entes de representación colectiva que recibieron el nombre de Comités Paritarios.

Sobre estos Comités, cabe decir que estuvieron integrados por obreros y patronos en igual proporción y que fueron presididos por personas designadas por el gobierno y dotadas con capacidad de veto ${ }^{5}$, algo que imponía una serie de limitaciones de las que el socialismo fue muy consciente, como queda claro en un texto propagandístico que se publicaría años después:

«Con todo, los Comités paritarios [Sic] tenían defectos casi fundamentales. El mayor de todos consistía en que el presidente era de libre elección del Gobierno. Y como el voto del presidente era decisivo, en muchas ocasiones perdían la batalla los obreros. Otras veces, el presidente dictaba un laudo favorable a las reivindicaciones obreras, y la empresa [...] se negaba a cumplirlo, sin que el Gobierno fuera con ella todo lo enérgico que debiera.» ${ }^{6}$

No obstante, dado que los trabajadores elegirían a sus representantes de entre los candidatos presentados por los sindicatos legales, los comicios para su designación eran una oportunidad única para que la UGT y el Sindicato Católico adquirieran adeptos allí donde se celebraran.

Este es el caso de los mineros onubenses y más concretamente de los de Riotinto,

\footnotetext{
${ }^{5}$ FERNÁNDEZ RIQUELME, Sergio: "Política, autoridad y trabajo. Eduardo Aunós y el estado corporativo en España", La Rąón Histórica-Revista Hispanoamericana de Historia de las ideas, No 10 (2010), pp. 17-31 $<$ http://www.revistalarazonhistorica.com/10-5/>.

PERFECTO GARCÍA, Miguel Ángel: "Política social y regeneracionismo en la dictadura de Primo de Rivera", Studia Zamorensia, No 1 (1994), pp. 223-242 <https://dialnet.unirioja.es/servlet/articulo?codigo=297496>.

${ }^{6}$ RAMOS OLIVEIRA, Antonio: Nosotros los marxistas. Lenin contra Marx, Madrid, Júcar, 1979, p. 171.
} 
que, como se ha adelantado, fueron derrotados después de la serie de huelgas acontecidas en 1920 y, salvo alguna excepción que se verá más adelante, vieron desaparecer al que había sido su sindicato de referencia durante la década anterior, la UGT.

Así las cosas, a principios de 1929, el gobierno y la Cámara Minera de Huelva iniciaron conversaciones para configurar lo que serían los Comités Paritarios de la minería de la provincia. La propuesta gubernamental consistía en la creación de tres de estos entes, integrando en uno de ellos a los representantes de las minas de Riotinto, explotadas desde 1873 por la Rio Tinto Company Limited 7 , y a los de Peña de Hierro, una explotación cuprífera de menor importancia que también estaba situada en la Cuenca Minera de Riotinto.

La patronal minera, por su parte, pretendía que la RTCL tuviera su propio Comité y que Peña de Hierro compartiera espacio con «las minas "Sultana", “Cala" y "Teuler".» El motivo de esta pretendida separación queda claro en una carta que el representante de la Cámara Minera de Huelva, Manuel Fernández Balbuena, remitió a César Madriaga, el Director General de Previsión y Corporaciones, a principios de 1929.

En ella, Fernández Balbuena recordaba que en Peña de Hierro seguía existiendo la UGT e incluso había, desde hacía años, una entidad de representación colectiva en la que se trataban los conflictos obreros con total aquiescencia de la dirección de la empresa. Aun así, lo más grave era que los mandos del sindicato y del mencionado Comité estaban en manos de Salvador Pino, un antiguo socialista que podría ver aumentado su "campo de acción" en el caso de que compartiera espacio con los obreros de otras minas, especialmente con los de Riotinto?.

De hecho, poco después de la carta que acaba de apuntarse, Fernández Balbuena se expresó en términos todavía más contundentes acerca del peligro que podría suponer un Salvador Pino al que se le suponía cierta aversión hacia la RTCL:

«Salvador Pino, es un propagandista de ideales no siempre compatibles con la marcha del trabajo en las minas. Y a estos ideales, mas [Sic] o menos intensamente sentidos, hay que unir el recuerdo, que para Pino tiene que ser inolvidable, de los kilometros recorridos por él, por carretera, en la grata compañia de la guardia Civil [Sic], viajes que realizó gracias a los esfuerzos perseverantes de nuestro antiguo amigo Browning ${ }^{10}$, y $\sin [\mathrm{Sic}]$ bien este nada tiene que ver con la Compañia, para Salvador Pino siempre sera la Compañia la que le obsequio con esos viajes de recreo.» ${ }^{11}$

\footnotetext{
${ }^{7}$ En adelante RTCL.

8 Archivo Histórico de la Fundación Río Tinto (AHFRT), Fondo Minas de Riotinto, leg. 1842, ds. 17-18.

9 AHFRT, Fondo Minas de Riotinto, leg. 1842, d. 9.

${ }^{10}$ Se refiere a Walter Browning, Director General de las minas de Riotinto durante las décadas de 1910 y 1920 y encargado de reprimir los movimientos huelguísticos de la primera de ellas, en los que Salvador Pino jugó cierto papel.

${ }^{11}$ AHFRT, Fondo Minas de Río Tinto, leg. 1842, ds. 34-35.
} 
Aun así, estos argumentos no debieron ser suficientes para convencer al titular de la Dirección General de Previsión y Corporaciones una vez que, el 9 de marzo de 1929, la Cámara Minera de Huelva se reunió e hizo dos nuevas propuestas de organización de los Comités Paritarios en las que las minas de Riotinto y Peña de Hierro compartían espacio, ante lo que el representante de la RTCL en aquella, José Sánchez Mora, se reservó el derecho de actuar en nombre de la empresa ${ }^{12}$.

Aquel mismo día, Manuel Fernández Balbuena escribió al representante de la RTCL en Madrid para comunicarle que inicialmente se englobaría a ambos establecimientos en el mismo ente, pero que existía «la promesa formal de que, transcurrido un corto periodo de uno o dos meses, al pedir Rio Tinto que se desglose de ese Comité, por las múltiples razones que en razón de esa petición se pueden alegar, se accederá inmediatamente a ello. Prefieren este sistema al de hacerlo abiertamente.» ${ }^{13}$

Por su parte y también el 9 de marzo de 1929, la dirección de la RTCL envió una carta al Ministro de Trabajo en la que insistía acerca de la idoneidad de contar con un ente de representación colectiva propio, al tiempo que le solicitaba una audiencia que desconocemos si llegó a producirse ${ }^{14}$.

En cuanto a la propuesta de la UGT, que apareció justó estos momentos, se hizo explícita en la única reunión con la Cámara Minera de Huelva de la que tenemos constancia, acontecida el 26 de enero de $1929^{15}$, y en el artículo $8^{\circ}$ de los estatutos de lo que será el Sindicato de Obreros Mineros de la Provincia de Huelva ${ }^{16}$, donde se defendía la creación de un solo Comité Paritario para toda la provincia.

Dado que las fuentes lo imponen, no podemos seguir refiriéndonos a las negociaciones. Pero lo que aquí interesa es que su resultado fue la creación de un solo Comité Paritario en el que la RTCL contaría con sección propia, lo que significó una solución intermedia entre las posturas del sindicato y la empresa aun siendo favorable a esta última, porque consiguió salvar su exclusividad respecto al resto de minas y evitar la "influencia" de Salvador Pino sobre sus trabajadores.

\footnotetext{
12 AHFRT, Fondo Minas de Riotinto, leg. 1842, ds. 36-39.

13 AHFRT, Fondo Minas de Riotinto, Leg. 1842, d. 42.

14 AHFRT, Fondo Minas de Riotinto, Leg. 1842, d. 43.

15 AHFRT, Fondo Minas de Riotinto, leg. 1842, d. 14.

16 AHFRT, Fondo Minas de Riotinto, leg. 1843, ds. 96-107.
} 


\section{Ramón González Peña y la fundación del Sindicato Minero de Huelva}

Antes de que todo esto ocurriera, en septiembre de 1928, había tenido lugar el V Congreso de la Federación de Obreros Mineros, en el que se nombró a Manuel Llaneza presidente y a Ramón González Peña secretario. También se exigió al gobierno la instauración de los Comités Paritarios de la minería, conforme al Decreto-ley del 26 de noviembre de $1926^{17}$, y se aprobó la puesta en marcha de una campaña propagandística en todas las cuencas mineras, especialmente en aquellas en que «no existe organización obrera, y que por el número de trabajadores merecen y es de necesidad incluirlos en nuestra Federación». ${ }^{18}$

La propaganda empezaría en Peñarroya, a donde deberían desplazarse González Peña y a un sindicalista de Vizcaya con el objeto de realizar «cierta gestión» en la Federación Regional de Sindicatos ${ }^{19}$. Después se trasladaría a las minas onubenses:

«Terminada su misión [en Peñarroya], González Peña regresó a Mieres, hasta que, de acuerdo con Llaneza y sus camaradas de la Federación, se trasladó a la provincia de Huelva, donde la organización obrera minera necesitaba una profunda reorganización. En la cuenca de Riotinto, Peña encontró auxiliares de primera categoría, alguno de ellos oriundo de Elche, con el ideal socialista metido en sus entrañas, como José Serrano ${ }^{20}$, que fue alcalde en Nerva y ha sido fusilado por los falangistas.» ${ }^{21}$

Efectivamente, eran los últimos días de 1928 cuando empezaron los actos propagandísticos y fue poco después, ya en 1929, cuando el secretario de la Federación

\footnotetext{
17 Aunque la campaña por su instauración en la minería empieza en este momento, la verdad es que la UGT llevaba promoviendo los Comités Paritarios desde finales de 1926, como queda claro en el informe presentado por Saborit al Congreso de la UGT que tuvo lugar del 10 al 15 de septiembre de 1928 y fue aprobado por amplia mayoría. El documento se reproduce en su integridad en ALQUÉZAR, Ramón y TERMES, Josep: Historia del Socialismo español. Vol 2 (1909-1931), Conjunto Editorial, Barcelona, 1989, pp. 316-319.

18 "V Congreso de la Federación de Obreros Mineros", El Socialista, 18 de septiembre de 1928.

${ }^{19}$ Ibidem.

${ }^{20}$ En realidad, se refiere a Antonio Serrano, que fue el primer alcalde republicano de Nerva y quien, junto a González Peña, firmó los Estatutos del Sindicato Minero en calidad de miembro del Comité ejecutivo. Antes de esto, en la década de 1910, también había sido uno de los principales impulsores del sindicalismo socialista y ello le valió el destierro de la Cuenca Minera de Riotinto.

AHFRT, Fondo Minas de Riotinto, leg. 1843, ds. 96-107.

FERNÁNDEZ DÍAZ, Rafael y ROMÁN GONZÁLEZ, Juan Francisco: Alcaldes de la Villa de Nerva. 1885 2002, Área de Cultura del Ayuntamiento de Nerva, 2002, pp. 125-128.

Fundación Pablo Iglesias: "Serrano Sánchez, Antonio", Diccionario biográfico del socialismo español, s.f. $<$ http://www.fpabloiglesias.es/archivo-y-biblioteca/diccionario-biografico/biografias/6917_serrano-sanchezantonio>.
}

LUNAR LÓPEZ, Félix: A cielo abierto. De Riotinto a Norteamérica, Huelva, Eds. Consulcom, 2011, p. 216.

${ }^{21}$ SABORIT, Andrés: Asturias y sus hombres, Tolouse, Ediciones UGT-SIOL, 1964, p. 178. 
minera de la UGT se instaló en Huelva para poner en pie el Sindicato ${ }^{22}$, lo que queda confirmado en base a las primeras palabras del artículo de Salvador Pino que fue publicado el $1^{\circ}$ de mayo de 1930 :

«Hace poco más de un año se cruzaron unas cartas, se emitieron opiniones, se señalaron fechas y por último en Nerva, burlando la oficiosidad de unos pobres diablos nos reunimos en el escondido rincón de un café nueve hombres. Era de noche, había poca luz y a pesar de esto no estábamos todos conformes en que se nos pudiera reconocer desde la calle. Estábamos en el corazón de Riotinto.

Peña nos habló, Peña era en aquel momento la Unión General de Trabajadores y el Partido Socialista. Los obreros organizados por conducto de su representante, nos daban la mano, nos invitaban a incorporarnos a su glorioso ejército, se constituyó el Sindicato y un año después somos más de 10.000 los soldados que enarbolan la bandera roja en esta provincia.» 23

Aparte de González Peña y los viejos socialistas que, como Antonio Serrano o Salvador Pino, secundaron su labor desde las minas onubenses, el propio Manuel Llaneza también jugó un papel importante en la reaparición del sindicalismo minero en el suroeste peninsular. Todo ello pese a que, justo en estos momentos, estaba desarrollando una intensa labor en su Asturias natal, por ejemplo, consiguiendo el impuesto sobre el carbón que sirvió para financiar el orfanato minero ${ }^{24}$.

La presencia de los dos propagandistas en las minas de Huelva, como quizá pueda imaginarse habiéndose visto la caracterización que Fernández Balbuena hizo de Salvador Pino, alertó de inmediato a unas compañías que, desde luego, no veían con buenos ojos que sus obreros se organizasen. No en vano, tanto el representante legal de la RTCL en Huelva, José Sánchez Mora, como algún miembro de la dirección de la Sociedad Francesa de Piritas de Huelva, que explotaba las minas de Valdelamusa (Cortegana), se expresaron en términos más que contundentes contra Llaneza.

22 ARENAS POSADAS, Carlos: Empresa, mercado, mina y mineros. Río Tino (1873-1936), Servicio de publicaciones de la Universidad de Huelva, 2000, p. 271.

MENÉNDEZ GARCÍA, Juan José: Ramón González Peña. "Generalisimo de la revolución", Gijón, Silverio Cañada Editor, 2009, p. 113., ubica su llegada a Huelva en 1927-1928 y COLLIER, George A.: Socialistas en la Andalucía rural: Los revolucionarios ignorados de la Segunda República, Huelva, Rubí, Anthropos, UGT Andalucía y Diputación Provincial de Huelva, 1997, p. 75., la inserta dentro de la campaña emprendida cara a las elecciones al Comité paritario de la minería a principios de 1929.

${ }^{23}$ PINO, Salvador: "El 1º de Mayo de 1930 en la provincia de Huelva", El Fraternal, Huelva, No 2, 1 de mayo de 1930.

Se trata del periódico del PSOE y la UGT onubenses, que sólo se publicó entre abril de 1930 y noviembre de 1931. Todos los números que hemos podido consultar están en AFRT, Fondo Minas de Rio Tinto, leg. 1809.

${ }^{24}$ MUÑIZ SÁNCHEZ, Jorge: "Encontrando el norte. Manuel Llaneza y la influencia francesa en el sindicalismo español de principios del s. XX", Hispania. Revista Española de Historia, vol. LXIX, No 233 (2009), pp. 793-820, p. 811, <http://hispania.revistas.csic.es/index.php/hispania/article/view/121/123>. y SABORIT, Andrés: óp. cit., pp. 219-220. 
En el primer caso, la opinión se vertió en una carta privada en la que se aseguraba que el líder ugetista había logrado fundar una nueva organización «que estará presta para luchar contra las Empresas, con el perjuicio a esta región. ${ }^{25}$ El representante de la Compañía Francesa de Piritas de Huelva fue mucho más explícito al expresarse, haciéndolo además en el seno de la Cámara minera y, obviamente, para un público algo más amplio ${ }^{26}$. Merece la pena darle la palabra para terminar de ilustrar la reacción de la patronal minera a la reaparición del socialismo:

«Hoy en día se está haciendo una organización obrera en manos de Llaneza, éste la utiliza como instrumento para cotizar su influencia cerca del gobierno. Me consta que éste así lo aprecia y entiende que es útil que una persona que, como Llaneza esté en contacto con el Gobierno y con los obreros, para poder manejarlos según las circunstancias aconsejen, circunstancias que, naturalmente, el Secretario de la Federación aprovecha en su ventaja, pero probablemente con daño nuestro.» ${ }^{27}$

La forma que la patronal de la mina de Valdelamusa proponía para enfrentarse al Sindicato Minero no era otra que la de hacer un frente común y, como según ella habían hecho los socialistas, utilizar la prensa a su favor. Pero, además de la propaganda, las compañías mineras tenían a su disposición todo un abanico de formas para intentar impedir que sus trabajadores se organizaran, algunas de ellas puestas en marcha desde hacía décadas, como la vigilancia policial o el control de las instituciones municipales ${ }^{28}$. Por otra parte, en el caso de Riotinto, se refundó un Sindicato Católico al que nos referiremos más adelante.

Más allá de a la oposición empresarial, González Peña y sus colaboradores también tuvieron que enfrentarse a quienes los criticaban por tratar de ocupar cargos en los entes de la dictadura. Obviamente, estos últimos debieron ser los antiguos cenetistas y los escasos adeptos al PCE que residían en la provincia, a quienes el líder minero respondió en un mitin celebrado en febrero de 1929 diciéndoles que el intervencionismo era la táctica de la UGT y que seguiría siéndolo en el futuro, por lo que no cabían ataques en este sentido ${ }^{29}$.

\footnotetext{
25 AHFRT, Fondo Minas de Riotinto, leg. 1842, ds. 167-168.

${ }^{26}$ Aunque las actas de las reuniones de la Cámara minera no contienen las intervenciones, sino que se trata de "actas de acuerdo", en el fondo de la Compañía Francesa de Piritas de Huelva se halla lo que podría ser una copia de la intervención de su representante en una reunión que debió celebrarse en marzo o abril de 1929, porque hace referencia a la comisión ministerial que debía dictaminar acerca de las condiciones de vida de los mineros de Tharsis.

La primera noticia acerca de tal comisión es del 30 de abril de 1929, lo que no es óbice para que la patronal minera estuviera previamente informada de las decisiones tomadas en Madrid referentes a su sector.

"El trabajo y la vida de los obreros en las minas de Tharsis", $A B C$, Madrid, 30 de abril de 1929.

27 AHFRT, Fondo Sociedad Francesa de Piritas de Huelva, leg. 71.

${ }^{28}$ Véase al respecto PEÑA GUERRERO, Ma Antonia: "Caciquismo y poder empresarial. El papel político de las compañías mineras en la provincia de Huelva (1898-1923)", Trocadero. Revista de historia moderna y contemporánea, $\begin{array}{llll}\mathrm{N}^{0} & 5 & \text { (1990), } & \text { pp. }\end{array}$ $<$ https://rodin.uca.es/xmlui/bitstream/handle/10498/9252/17216515.pdf? sequence=1\&isAllowed=y $>$.

${ }^{29}$ PINO, Salvador: "Conferencias de González Peña/Nerva", El socialista, 6 de febrero de 1929. El acto tuvo lugar el día 4 y el mismo día hubo otro en El Campillo.
} 
En otras palabras, que la entidad que se estaba formando tenía como fin intervenir en los conflictos laborales a través de la participación en los Comités Paritarios y, con ello, dejaba a un lado la táctica "de choque" que había predominado durante la década anterior. Esta última, la "táctica de choque", había dado lugar a multitud de huelgas encabezadas por una serie de hombres que ahora serán tachados de inconscientes, como se desprende de lo dicho por González Peña en un acto público celebrado en febrero de 1929:

«Queremos una organización fuerte, disciplinada, y trataremos de hacerla en esta provincia; pero no deseamos que los obreros vengan en aluvión, arrastrados por un entusiasmo que se apague al choque con la dura realidad. No queremos volver a pensar en vuestras luchas pasadas; pero sí queremos ¿cómo no?, que los obreros de Riotinto se incorporen a sus compañeros de otras regiones, para que todos unidos podamos dar una seria personalidad jurídica real y efectiva al Sindicato, que es, en fin [Sic] de cuentas, de donde ha de salir vuestra representación en los Comités Paritarios.

Queremos dar vida al Sindicato Minero de Huelva, que estará integrado por tantas Secciones como podamos crear, y ellas serán las que designen su Comité Ejecutivo, que en todo momento velará por el interés de sus trabajadores, sin que tenga facultades nunca para llevarlos a movimientos irreflexivos y perjudiciales.» ${ }^{30}$

Además, esta nueva táctica quedará sancionada por los estatutos del sindicato ${ }^{31}$, que fueron presentados en el Gobierno civil el 16 de febrero de 1929 y dados por válidos el 24 de aquel mismo mes ${ }^{32}$, lo que viene a ser una prueba más de que de lo que se trataba era de participar en las elecciones del Comité Paritario una vez que el 26 de abril se abrió el último plazo de inscripción en el Censo electoral del Ministerio de Trabajo cara a tal evento ${ }^{33}$.

En efecto, el reglamento del Sindicato establecía un modelo de organización centralizado tanto en lo económico, pues las secciones sólo podrían contar con los fondos necesarios para su funcionamiento cotidiano, como en lo estrictamente sindical, al establecer un mecanismo que primaba la decisión del Comité Ejecutivo sobre las tomadas por las bases. En este último sentido, conviene señalar que sólo podría declararse una huelga en los casos en los que las vías de negociación formal se hubieran agotado, después de que lo decidiese un Congreso extraordinario y tras un referéndum en el que dos terceras partes de los afiliados se posicionaran a favor de hacerlo ${ }^{34}$.

\footnotetext{
${ }^{30}$ Ibidem.

31 AHFRT, Fondo Minas de Riotinto, leg. 1843, ds. 96-107.

32 Archivo Histórico Provincial de Huelva (AHPH), leg. 7026, Registro N 745.

33 "Decreto No 572", Gaceta de Madrid, 116, 26 de abril de 1929, p. 116. Recuperado de internet (https://www.boe.es/datos/pdfs/BOE//1929/116/A00510-00510.pdf).

${ }^{34}$ AHFRT, Fondo Minas de Riotinto, leg. 1843, ds. 96-107. Artículos 53 a 55.
} 
«Cuando despues [Sic] de verificado el referéndum apareciese la mayoria que en el articulo 55 se determina, esta tendra que ser secundada por todos los Comités y afiliados, encauzando la lucha por los derroteros que a la organizacion segun las circunstancias conviniera, procurando aprovechar cuantas coyunturas favorables se les presenten, a fin de hallar una fórmula de avenencia con los patronos. $»^{35}$

De esta forma, las secciones locales se veían despojadas de la posibilidad "legal" de emprender paros por sí mismas y, al menos en teoría, la Federación minera se evitaba tener que afrontar brotes huelguísticos como los de 1913, cuando los obreros de un departamento iniciaban la lucha y esta se extendía rápidamente para terminar por agotamiento.

Ni que decir tiene, que la principal razón de que se optara por un modelo organizativo como el que acaba de describirse hay que buscarla en la necesidad de adaptar el sindicato a la legalidad de la dictadura. Sin embargo, hay otro motivo de peso a la hora de generar tal realidad: los protagonismos de Llaneza y González Peña.

Para entenderlo, cabe recordar que Llaneza era partidario de un sindicalismo que, por hacerlo de algún modo, podríamos denominar como "moderado" en la medida de que buscaba evitar huelgas siempre que fuera posible.

$\mathrm{Al}$ respecto, se hace necesario mencionar el enfrentamiento de Llaneza con Facundo Perezagua acontecido entre 1913 y 1915, cuando el segundo se unió a los organizadores del primer Sindicato Minero de Riotinto y puso en marcha una táctica ofensiva que, en el plano orgánico, se saldó con la salida de los asturianos de la Federación Nacional de Obreros Mineros de España ${ }^{36}$. También hay que recordar la escisión que tuvo lugar en el Sindicato Minero asturiano en los primeros años 20, momento en el que los comunistas y los anarcosindicalistas mantuvieron un importante pulso con la dirección de Llaneza y llegaron a fundar el Sindicato Único de Mineros de Asturias ${ }^{37}$.

Así pues, no siendo necesario insistir en la concepción sindical de Ramón González Peña por entenderla lo suficientemente ilustrada en la intervención que ha quedado transcrita, pensamos que es posible concluir que el Sindicato de Obreros Mineros de Huelva de los últimos momentos de la dictadura era una entidad pragmática, centralizada en la toma de decisiones o el funcionamiento y poco dada a esas huelgas "irreflexivas" que ya hemos visto criticadas en las palabras de su propio organizador.

\footnotetext{
35 Ibídem. Artículo 56.

${ }^{36}$ FASEFELDT, Henrike, "Del mundo de los oficios a la lucha de intereses: la UGT, 1888-1923", Ayer, No 54 (2004), pp. 71-96, pp. 85-86

<https://www.ahistcon.org/PDF/numeros/ayer54_125FundacionPSOE_Forcadell.pdf>.

37 ÁLVAREZ, Ceferino: "El Sindicato Único de Mineros de Asturias 1922-1935”, Espacio, Tiempo y Forma, Serie V, H." Contemporánea, No 15 (2002), pp. 279-315. <https://doi.org/10.5944/etfv.15.2002.3070>.
} 
Aunque quede fuera del marco temporal que nos hemos marcado, creemos que procede decir que ello vinculará directamente al socialismo minero de Huelva con su homónimo estatal durante los próximos años, pasando ambos prácticamente por las mismas etapas y sirviendo de sostén a la II República durante su primer bienio.

No en vano, tal pragmatismo fue elogiado por uno de los primeros gobernadores civiles de la II República, Braulio Solsona, en un pequeño anecdotario en el que reconocía que sin el espíritu de sacrificio de la UGT el Gobierno republicano-socialista habría tenido que enfrentarse a innumerables conflictos en las minas de Huelva ${ }^{38}$.

\section{La evolución afiliativa del Sindicato Minero en la Cuenca Minera de Riotinto}

Ya fuera pese a la moderación que acaba de comentarse o gracias a ella, lo cierto es que la refundación de la UGT en las minas onubenses tiene que ser considerada como un éxito rotundo que, aunque ya haya sido ilustrado en las palabras de Salvador Pino, tiene que mostrarse con la mayor precisión posible.

Aun así, a día de hoy sólo hemos conseguido obtener los datos referentes a dos de sus secciones, la de Nerva y la de El Campillo, pero son las que encuadraban a todos los afiliados del Riotinto de la época y, por tanto, las más representativas. Esto ocurrirá hasta que, después del cambio de régimen, la organización se extienda por todos los municipios, lo que queda ilustrado en base a que fue la Agrupación Socialista de Nerva quien presentó la candidatura a las elecciones del 12 de abril de 1931 en Minas de Riotinto ${ }^{39}$, donde las organizaciones obreras vieron imposibilitada su labor por ser el pueblo propiedad exclusiva de la RTCL ${ }^{40}$.

Para entender este hecho hay que decir que las minas de Riotinto eran las más importantes de la provincia, lo que traía consigo que los pueblos que dependían de ellas fueran los más populosos y posibilita que puedan ser tomadas de ejemplo. Aun así, conviene advertir que somos conscientes de que en cada caso debieron operar ritmos y/o actores distintos, por lo que no pretendemos extrapolar el particular a toda la provincia más que en la medida de lo posible.

\footnotetext{
38 SOLSONA, Braulio: El señor gobernador, Barcelona, Editorial Leyes, 1934, p. 91.

39 AHFRT, Fondo Minas de Riotinto, leg. 1843, d. 585.

Es posible que en el caso de Zalamea la Real ocurriera un fenómeno similar en la medida de que El Campillo era su aldea y fue el único pueblo de la comarca en el que la Conjunción Republicano-Socialista contenía elementos de la primera cultura política. VÁZQUEZ LAZO, José Manuel: Zalamea Republicana, Diputación Provincial de Huelva, 2005, pp. 63-76.

${ }^{40}$ En consecuencia, era el lugar de residencia del Staff británico de la RTCL, de los mandos medios de la mina, de los ingenieros y de los trabajadores más "afines" a la empresa. Todo ello dio lugar a un nivel de control social que en Nerva y El Campillo se verá menguado, pues sus términos municipales conservaban cierta independencia respecto a las minas. Esto será matizado tras la proclamación de la II República. GIL VARÓN, Luis: Minería y migraciones. Río Tinto 1873-1973, Córdoba, ed. del autor, 1984, pp. 50-59.
} 
Por último, respecto a la fuente en sí, hay que afirmar que no se trata de datos emanados del propio Sindicato Minero, sino de cifras recogidas por los informantes que la RTCL tenía repartidos por los municipios e incluso infiltrados en las organizaciones obreras ${ }^{41}$. $\mathrm{Ni}$ que decir tiene que ello constituye un hándicap, dado que es indudable que la documentación "oficial” sería infinitamente más precisa que la que nos vemos obligados a utilizar $^{42}$, pero creemos que el interés de la empresa por mantenerse informada acerca del avance organizativo de sus trabajadores es más que suficiente como para que la documentación que manejaba tenga que ser considerada más o menos válida, al menos, para este caso.

Hechas estas salvedades, a las que hay que sumar el hecho de que los documentos sólo abarcan el periodo que va desde mayo de 1929 hasta octubre de 1930, ha llegado el momento de mostrar cuál fue la evolución afiliativa del Sindicato Minero en Nerva y El Campillo:

\section{Gráfico 1}

\section{Afiliados al Sindicato Minero en Nerva y El Campillo (mayo de 1929-octubre de} 1930)

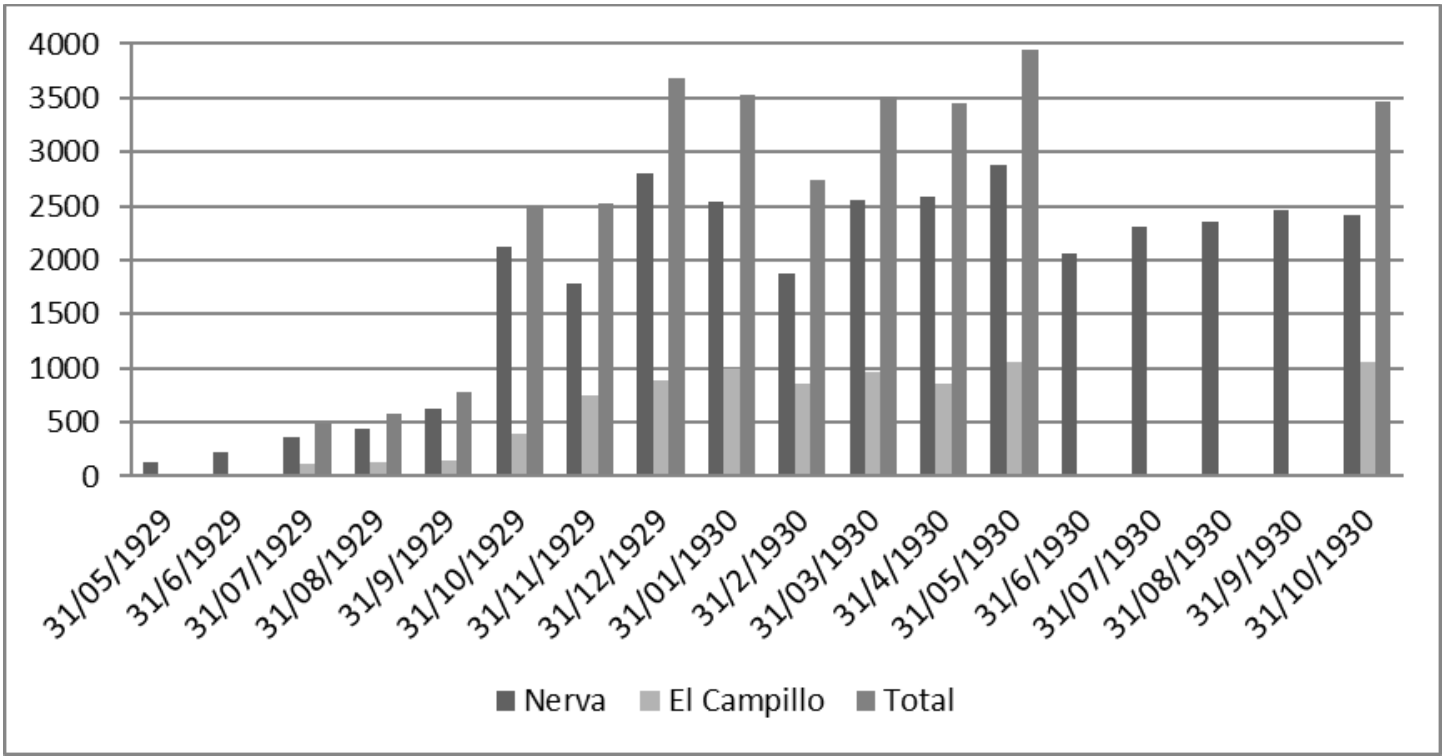

AHFRT, Fondo Minas de Riotinto leg. 1843, ds. 546-549 y AHFRT, Fondo Minas de Riotinto, leg. 1843, ds. 552-553

${ }^{41}$ AHFRT, Fondo Minas de Riotinto, leg. 1843, d. 561.

${ }^{42}$ Los locales de las organizaciones obreras fueron saqueados tras la llegada de los sublevados y parece que la documentación que contenían ardió. 
Como puede verse, en la mencionada evolución afiliativa se observan dos periodos bien diferenciados: el que va desde principios de 1929 hasta el verano de aquel año, en el que se da un crecimiento más o menos sostenido pero de poca intensidad, y el que se inicia en septiembre-octubre y dura hasta el final del lapso recogido en los datos, donde los cotizantes sobrepasan a los dos millares para el caso nervense.

El salto de octubre de 1929, podría resultar evidente, debió estar causado por la proximidad de las elecciones y el inherente incremento de la propaganda. Sin embargo, el 1 de septiembre de 1929, un guardia de la RTCL residente en Nerva informó acerca de la visita a la capital onubense de un dirigente local «haber [Sic] si podía venir un charlatan [Sic] y le dijeron que por orden del ministro esta prohibida la propaganda en la provincia de Huelva. Ha sí [Sic] que con unas cosas y otras se nota mucha frialdad.» ${ }^{43}$

Pese a la prohibición anunciada, cuatro días más tarde hacía su aparición un viejo conocido de los mineros: el mismo Agustín Marcos que, junto a Eladio Fernández Egocheaga, había organizado el sindicato de la década de $1910^{44}$. Puede que su sola presencia sirviera de estímulo para las afiliaciones, pero lo cierto es que fue sometido a una intensa vigilancia por parte de la RTCL y es muy posible que sus contactos con los trabajadores se vieran profundamente limitados.

No en vano, pocos días después de su llegada, otro de los informantes de la RTCL afirmaba que Marcos se mostraba desilusionado e incapaz de construir una organización sólida, por lo que anunciaba su próxima marcha hacia Madrid ${ }^{45}$. Partió a finales de mes, según los informes de la empresa, para unirse a Saborit, tratar de entrevistarse con un ministro y solicitar el permiso para la celebración de un acto. También pretendían convencer al Gobierno de la virtualidad del Sindicato Católico con el objeto de impedir su participación en las elecciones ${ }^{46}$.

En aquel mismo informe, se mencionaba la pérdida de esperanza de ganar las elecciones que sufrían los militantes del Sindicato Minero, dado que había una buena porción que no pagaba las cuotas y otra que no votaría por estar compuesta por menores de edad o personas con miedo a perder el trabajo.

Aun así, parece que Agustín Marcos consiguió parte de sus propósitos y el 6 de octubre, después de que los dirigentes locales se esforzaran por encontrar un

\footnotetext{
43 AHFRT, Fondo Minas de Riotinto, leg. 1843, d. 191.

${ }^{44}$ AHFRT, Fondo Minas de Riotinto, leg. 1843, d. 193.

45 AHFRT, Fondo Minas de Riotinto, leg. 1843, d.152.

46 AHFRT, Fondo Minas de Riotinto, leg. 1843,d.158.
} 
emplazamiento $^{47}$, se celebró un mitin en la plaza de toros de Nerva ${ }^{48}$. En aquel acto, al que acudieron unas mil trescientas personas, Marcos empleó un tono duro tanto hacia la empresa como hacia un Sindicato Católico que acababa de entrar en escena. También introdujo un elemento que diferenciaba su discurso del de González Peña, el de considerar las huelgas de la década de 1910 como algo digno de ser recordado:

«Aqui [Sic] en esta plaza de toros lo mismo que en los años 13 al 16 se discutiran y aprobaran todos los muchos asuntos de gran importancia que en beneficio vuestro, obreros de Rio Tinto en esta plaza trataremos, y con eso al mismo tiempo recordaremos aquellas asambleas hermosísimas en la que a cada momento poníais de manifiesto que erais hombres, porque [de] eso no me cabe la menor duda, esta tarde los estais demostrando.» ${ }^{49}$

Detrás de esta diferencia de visión acerca de las luchas pasadas, obviamente, podría estar el protagonismo que el propio Marcos había ostentado, como también lo estarían la cercanía de las elecciones y la inherente necesidad de intensificar la propaganda tanto en el fondo como en las formas.

No en vano, en aquella ocasión se utilizaron dos recursos que, aunque someramente, tenemos que comentar:

1. Por un lado, Agustín Marcos contrapuso la masculinidad de los mineros ugetistas a la de los sindicalistas católicos, que era cuestionada.

Valga decir que este recurso no hace sino poner de manifiesto la concepción de masculinidad que dominaba en la España de la época, también entre el movimiento obrero, y que tiene que ser considerado como algo fundamental en la construcción de las identidades políticas, dado que la identificación de la "hombría" con los huelguistas lleva implícita una interpretación en la que la función social de cada persona viene determinada por el sexo y, en consecuencia, la de los hombres pasaba por luchar frente a las injusticias, mientras que las mujeres tendrían un papel pasivo $\mathrm{o}$, si se quiere, complaciente para con el poder ${ }^{50}$.

\footnotetext{
${ }^{47}$ Intentaron conseguir algún local privado, como la sede de la Juventud Cultural, pero no tuvieron éxito por la acción de la RTCL. En el documento que queda reseñado se relata, con todo lujo de detalles, una reunión mantenida entre el presidente de esa entidad y un alférez de la Guardia Civil acerca del encuentro del primero con los líderes del sindicato nervense.

AHFRT, Fondo Minas de Riotinto, leg. 1843, ds. 219-234.

48 AHFRT, Fondo Minas de Riotinto, leg. 1843, ds. 210-213.

${ }^{49}$ Ibidem.

${ }^{50}$ Véanse al respecto: ARESTI, Nerea: "La historia de las masculinidades, la otra cara de la historia de género", Ayer, no 117, 2020, pp. 333-347. y ARESTI, Nerea: "Masculinidad y nación en la España de los años 1920 y 1930”, Mélanges de la Casa de Velázquez, nº 42, 2, 2012,, pp. 55-72.

$<$ https://journals.openedition.org/mcv/4548>.
} 
Es decir, que dentro de la contraposición nosotros-ellos/bondad-maldad, que podría ser usual en la propaganda destinada a construir un nuevo sujeto político, se incluyó un recurso de carácter sexista que no podemos pasar por alto, dado que se trata de algo muy significativo en lo que respecta a la caracterización de la sociedad y de los líderes sindicales del momento.

2. Por el otro, recurrió a una xenofobia empresarial que era frecuente entre los oradores foráneos que llegaban a la comarca, independientemente de filiaciones políticas y sindicales.

Sobre este asunto, cabe decir que Salvador Seguí visitó la comarca durante las huelgas de 1920 y llegó a afirmar que el conflicto estaba motivado por la intromisión británica en la Cuenca Minera de Riotinto ${ }^{51}$, algo muy parecido a lo que criticó Ramiro de Ledesma en el primer número de Nuestra Revolución ${ }^{52}$ o a las palabras pronunciadas por el Secretario Provincial de FET y de las JONS en un acto que tuvo lugar en septiembre de 1937, en el que se dirigió a los mineros diciéndoles que si no se hubieran acercado a las izquierdas, y se hubiera producido la unidad nacional que propugnaba Franco, las minas de Riotinto no habrían servido para enriquecer a Gran Bretaña ${ }^{53}$.

Pese a estos ataques, que también afectaron a los guardias de la RTCL y a las organizaciones que esta fomentaba, el régimen dictatorial era bien considerado, pues había legislado en lo referente a las relaciones laborales y ello implicaría el fin de la Agencia de Trabajo y las contrataciones por recomendación:

«Nosotros los socialistas enemigos del regimen [Sic] actual tenemos sin embargo, que dar las gracias a este Gobierno que ha sabido dar leyes tan beneficiosas para los obreros como son las del Comité Paritario, asi hay que reconocerlo. Yo también quisiera obreros de Rio Tinto darle las gracias desde aquí a la Compañía de Rio Tinto si algo bueno hubiera hecho; pero siento no poderselas dar por que [Sic] la Compañía de Rio Tinto es muy mala.» ${ }^{54}$

\footnotetext{
51 "El conflicto minero de Riotinto. Habla el «Noi del Sucre»", $A B C$, Madrid, 21 de noviembre de 1920.

52 LEDESMA RAMOS, Ramiro: “El capitalismo extranjero en España”, Nuestra Revolución, 11 de julio de 1936. Denuncia el enriquecimiento de la RTCL a costa de los españoles y por exportar la mayor parte del mineral en crudo, por lo que sugiere la nacionalización de las minas.

53 "Nacionalsindicalismo. En Río Tinto y Nerva, se celebran actos de propaganda, en los que el camarada Pardo, nuestro Jefe Provincial, hizo llegar al pueblo la voz de Falange”, Odiel, Huelva, 21 de septiembre de 1937.

54 AHFRT, Fondo Minas de Riotinto, leg. 1843, ds. 210-213.
} 
El resultado de aquel mitin y del celebrado en El Campillo ${ }^{55}$ puede quedar ilustrado en el gráfico de más arriba, pero conviene añadir que, el 11 de octubre, uno de los informantes de Nerva afirmó que en sólo dos días el sindicato había sumado 120 afiliados ${ }^{56}$. El mismo día, otro guarda de El Campillo decía que allí sobrepasaban los $450^{57}$. Más ilustrativo aún es el informe de Benito León del 9 de octubre, en el que se puede leer que «debido a la asamblea del domingo se están apuntando en casa de Serrano con descaro y sin temor a nada. El asunto marcha muy mal. $\gg^{58}$

Y, a ojos de la RTCL, el "asunto" debía marchar realmente mal, porque, el 14 de octubre, un grupo de trabajadores dirigió una misiva a Primo de Rivera en la que aseguraban que las elecciones sólo servirían para el provecho de algunos «advenedizos» llegados de fuera, por lo que proponían su anulación ${ }^{59}$.

\section{La opción sindical de la Rio Tinto Company Limited: el Sindicato Católico}

Aparte de enviar esta carta, que por cierto estaba firmada por obreros de todos los oficios y poblados, la RTCL puso en marcha el ya mencionado Sindicato Católico, que había existido en 1919-1920 y contado con secciones en Minas de Riotinto, Nerva y Zalamea la Real $^{60}$.

Las primeras noticias de su fundación son de muy poco antes de que se produjeran el mitin y las reacciones a las que acabamos de referirnos. Se trata de una carta firmada por el antiguo Presidente de la sección riotinteña del Sindicato, que iba dirigida a su homónimo estatal, Vicente Madera Peña ${ }^{61}$, y está fechada el 5 de octubre ${ }^{62}$. En ella, el riotinteño se preocupaba por la situación legal de la entidad e informaba de que, pese a no haber mantenido el contacto en casi diez años, el Sindicato Católico de las minas de Riotinto seguía existiendo, de que mantenía a las mismas personas en los puestos directivos y de que contaba con quinientos afiliados. También solicitaba reglamentos, hojas de afiliación y pedía que se les redujera la cuota para poder alcanzar los dos mil asociados.

\footnotetext{
55 AHFRT, Fondo Minas de Riotinto, leg. 1842, d. 164.

56 AHFRT, Fondo Minas de Riotinto, leg. 1843, d. 163.

57 AHFRT, Fondo Minas de Riotinto, leg. 1843, d. 190.

58 AHFRT, Fondo Minas de Riotinto, leg. 1843, d. 194.

59 AHFRT, Fondo Minas de Riotinto, leg. 1843, ds. 236-238.

${ }^{60}$ RUIZ SÁNCHEZ, José Leonardo: "La Acción Social en la Provincia de Huelva (1902-1922). Algunas consideraciones para su estudio y comprensión”, Huelva en su historia, 5, 1994, pp. 457-484. <http://rabida.uhu.es/dspace/bitstream/handle/10272/2965/b1513961x.pdf?sequence=1>, p. 479.

${ }^{61}$ Era familiar de Ramón González Peña y el único dirigente del Sindicato Católico desde su fundación hasta el franquismo, cuando pasará a integrarse en FET y de las JONS y a ostentar varios cargos. BURGOS FERNÁNDEZ, Ernesto, "Vicente Madera, del amarillo al azul Mahón”, La nueva España, 15 de enero de 2013. $<$ https://www.lne.es/cuencas/2013/01/15/vicente-madera-amarillo-azul-mahon/1354135.html>.

${ }^{62}$ AHFRT, Fondo Minas de Riotinto, leg. 1844, d. 135.
} 
La respuesta de Madera Peña, que data de sólo tres días después, indicó que el Sindicato Católico de Riotinto aparecía en el censo con mil quinientos adherentes facultados para votar en las próximas elecciones. Para evitar fraudes, recomendaba que se requiriera la presencia de delegados del alcalde en las mesas electorales. Y, por último, tranquilizaba a los riotinteños ante una eventual derrota, pues se había solicitado la presencia de las minorías en los Comités Paritarios y confiaba «en la justicia y en la energía y constancia con que pedimos en este caso.» ${ }^{63}$

En una carta posterior, Madera Peña informaba de que era imposible modificar el importe de las cuotas, pues era competencia del Congreso, pero que ello no era impedimento porque el Sindicato podría decir que las secciones de Riotinto estaban al día en todos los asuntos. Para seguir organizando el ente, el asturiano sugería que se imprimieran unos carnets sencillos y sellos con los que justificar las cotizaciones ${ }^{64}$. Esto último le fue solicitado a la Agencia de Trabajo de la RTCL, mientras se la instaba a no cobrar las afiliaciones hasta después de los comicios para evitar bajas ${ }^{65}$.

Por si la virtualidad del Sindicato Católico no estuviera lo suficientemente clara, citaremos la misiva que Madera Peña envió a los sindicalistas de Riotinto el 26 de octubre de 1929, en la que sugería a sus organizadores falsificar un libro de afiliados si no les era posible conseguir uno a tiempo para las elecciones y daba algún que otro consejo extra:

«Las secciones de nueva creación como la Vds. han fundado en Nerva, no pueden votar, pues para ello, necesitan inscribirse en el "Censo Electoral Social", mediante una serie de documentos que al efecto tienen y que oportunamente les indicaremos pero ahora no ha lugar.

Lo que deben hacer es asegurar los 1.500 votantes de la sección de Rio Tinto inscribiendo en esta a los de Nerva si no tienen bastantes socios en ella para sacar el número citado de votos. $\rangle^{60}$

Para llegar a los mil quinientos afiliados, la RTCL y el Sindicato Católico pusieron en marcha una campaña que, según la UGT, pasaba por coaccionar a los mineros en sus propias casas y en el centro de trabajo ${ }^{67}$. En esta labor, al parecer, estuvieron implicados tanto los guardas de la empresa como los capataces ${ }^{68}$, que no debieron tener mucho éxito porque

\footnotetext{
${ }^{63}$ AHFRT, Fondo Minas de Riotinto, leg. 1844, d. 134.

64 AHFRT, Fondo Minas de Riotinto, leg. 1844, d. 120.

65 AHFRT, Fondo Minas de Riotinto, leg. 1844, d. 107.

66 AHFRT, Fondo Minas de Riotinto, leg. 1844, d. 112.

${ }^{67}$ Por ejemplo, en MARCOS ESCUDERO, Agustín: “Las coacciones de la empresa de Rio Tinto”, El Socialista, 19 de octubre de 1929.

68 AHFRT, Fondo Minas de Riotinto, leg. 1844, ds. 129-130.
} 
algunos se quejaron de la poca efectividad a consecuencia de la acción de los socialistas ${ }^{69}$. Otra muestra son las listas de afiliados que se han conservado, en las que se pueden encontrar ochocientos dos hombres residentes en su mayoría en Minas de Riotinto, mineros de base o pertenecientes al cuadro técnico y administrativo y con una edad media de en torno a los cuarenta años ${ }^{70}$. Es decir, que por regla general eran los mismos que habían engrosado sus filas diez años antes.

\section{Los mineros se manifiestan: los resultados de las elecciones al Comité Paritario de} la minería onubense en las minas de Riotinto

Habiéndose visto cuáles fueron las opciones sindicales entre las que pudieron elegir los mineros de Riotinto en las elecciones de 1929, sólo queda decir que estas se celebraron en diciembre de 1929, después de varios aplazamientos, y que arrojaron unos resultados abrumadoramente favorables al socialismo, como puede comprobarse en el cuadro en el que la RTCL expresó el recuento de votos:

\section{Cuadro 1}

Resultado de las elecciones a los Comités paritarios de la minería en la Cuenca Minera de Riotinto

\begin{tabular}{|l|c|c|c|c|c|} 
& Socialistas & Adictos & En Blanco & Total & Censo \\
\hline Minas de Riotinto & 1.543 & 540 & 24 & 2.106 & 3.299 \\
\hline Nerva & 2.209 & 217 & 1 & 2.427 & 3.728 \\
\hline El Campillo & 708 & 22 & - & 730 & 1.086 \\
\hline Zalamea la Real & 492 & 52 & - & 544 & 938 \\
\hline TOTAL & $\mathbf{4 . 9 5 2}$ & $\mathbf{8 3 1}$ & $\mathbf{2 5}$ & $\mathbf{5 . 8 0 8}$ & $\mathbf{9 . 0 8 1}$ \\
\hline
\end{tabular}

Fuente: AHFRT, Fondo Minas de Riotinto, leg. 1842, ds.91-92

En efecto, partiendo de un 63,9\% de participación, el 85,3\% de la plantilla optó por la opción socialista y sólo el 14,3\% lo hizo por el Sindicato Católico, que en la documentación de la empresa aparece identificado como "adicto". Esto significa que el 54,5\% de los mineros

\footnotetext{
${ }^{69}$ AHFRT, Fondo Minas de Riotinto, leg. 1842, d. 129.

${ }^{70}$ Se trata de un total de quince listas, una por cada núcleo de población, que pueden encontrarse en AFRT, Fondo Minas de Riotinto, leg. 1842. No las citamos para evitar sobredimensionar innecesariamente el espacio a pie de página.
} 
de Riotinto se decantó por la opción encabezada por Ramón González Peña ${ }^{71}$ pese a la labor de la RTCL en su contra.

Estos mismos resultados, e incluso más favorables para las listas en las que hubiera presencia socialista, se repitieron en todas las elecciones que se celebraron en los años posteriores $^{72}$, lo que no deja de ser la mejor muestra de que la labor emprendida por los delegados de la Federación Sindical Minera de la UGT fue un éxito rotundo.

\section{Conclusiones}

La UGT reapareció en las minas de Riotinto en los últimos momentos de la dictadura de Primo de Rivera con un objetivo muy claro: el de participar en las elecciones al Comité Paritario de la minería onubense y ocupar los puestos de representación colectiva del colectivo minero.

Para hacerlo, envió a Huelva a los dos líderes del Sindicato Minero asturiano más destacados, Manuel Llaneza y González Peña, que pusieron en pie una organización que contrastaba con la existente en la década de 1910 pero era completamente afín a los postulados que defendían: centralización económica y organizativa, evitación de huelgas en pro del diálogo social y pragmatismo en el funcionamiento.

Pese a esta circunstancia, la patronal minera puso en marcha una serie de iniciativas que pasaron por la vigilancia activa de los socialistas, la propaganda y, en el caso de Riotinto, la recuperación de un Sindicato Católico que en el plano estatal estaba encabezado por un familiar directo de González Peña, lo que no puede dejar de ser señalado por su importancia simbólica.

No obstante, los resultados de la acción de los ugetistas fueron abrumadoramente favorables y consiguieron no sólo la mencionada representación en los comités paritarios, sino también asentar un sindicato cuya fuerza se ha visto representada en unos resultados electorales que se verán repetidos en todos y cada uno de los comicios subsiguientes, en los que las candidaturas en las que el PSOE tenga representación obtendrán siempre más del 80\% de los apoyos.

\footnotetext{
71 AHFRT, Fondo Minas de Riotinto, leg. 1842, ds.91-92.

La lista para la sección de las minas de Riotinto estaba compuesta por José Carabante Pérez, Ramón González Peña y Luciano López Martín. Sus suplentes serían Juan Manuel Romero Cordón, Juan García Bravo y Elías Palma Ortega. Los candidatos titulares a la sección del resto de las minas de la provincia fueron José Romero Báez, José Barba Delgado, Justo Pozas Delgado y Elías Palma Ortega, que serían suplidos por Benito Serrano Carrasco, Remigio Pozas Delgado, Antonio Blanco Jiménez y Ramón González Peña.

${ }^{72}$ GARCÍA GARCÍA, Cristóbal: Modernización politica y pervivencias caciquiles. Huelva, 1931-1936, Ayuntamiento de Huelva, 2001.
} 
Ello ubicó a los mineros de Huelva, y posiblemente a sus organizadores, en la primera fila del socialismo español, siendo indiscutible que su peso será determinante tanto en el seno de la Federación del gremio de la UGT como en el propio PSOE, lo que queda manifestado en el nombramiento de González Peña como primer Gobernador civil de la provincia tras la caída de la monarquía ${ }^{73}$ o en los resultados de las elecciones de 1933, a las que los socialistas fueron solos y únicamente resultaron vencedores en las circunscripciones de Madrid capital y la provincia onubense.

En definitiva, puede concluirse que la acción de González Peña en Huelva durante los últimos momentos de la dictadura, aun yendo encaminada a conseguir unos fines muy limitados, fue transcendental no solo para las zonas geográficas en las que se centró, sino también para las organizaciones socialistas del grueso del Estado, que a la altura de 1929 eran inexistentes en el suroeste español y a partir de su llegada se verán consolidadas de forma indiscutible.

73 “Ayer, día 14, ha quedado proclamada la República en España”, Diario de Huelva, 15 de abril de 1931. 